\title{
The missing fundamental in vowel height perception
}

\author{
RICHARD P. FAHEY and RANDY L. DIEHL \\ University of Texas, Austin, Texas
}

\begin{abstract}
Traunmüller (1981) suggested that the tonotopic distance between the first formant $\left(F_{1}\right)$ and the fundamental frequency $\left(F_{0}\right)$ is a major determinant of perceived vowel height. In the present study, subjects identified a vowel-height continuum ranging in formant pattern from $/ \mathbf{I} /$ to $/ \varepsilon /$, at five $F_{0}$ values. Increasing $F_{0}$ led to an increased probability of /I/ responses (i.e., the phoneme boundary shifted toward the / $\varepsilon$ / end of the continuum). Various conditions of filtering out the lower harmonics of the stimuli caused only marginal shifts of the phoneme boundary. The experiments provide evidence against interpretations of Traunmüller's (1981) results that claim that vowel height is determined by the distance between $F_{1}$ and the lowest harmonic that is present in the basilar membrane excitation pattern.
\end{abstract}

Early research on the acoustic properties of vowels established that the first formant frequency $\left(F_{1}\right)$ varies inversely with vowel height ${ }^{1}$ (Chiba \& Kajiyama, 1941/ 1958; Peterson \& Barney, 1952; Potter, Kopp, \& Green, 1947; Potter \& Steinberg, 1950), whereas fundamental frequency $\left(F_{0}\right)$ varies directly with vowel height (House \& Fairbanks, 1953; Lehiste \& Peterson, 1961; Peterson $\&$ Barney, 1952). Moreover, listeners are sensitive to both of these correlates: increasing $F_{1}$ decreases perceived height (Delattre, Liberman, Cooper, \& Gerstman, 1952; Miller, 1953), whereas increasing $F_{0}$ increases perceived height (Potter \& Steinberg, 1950; Miller, 1953).

More recently, Traunmüller (1981) suggested that the appropriate cue for vowel height is the tonotopic distance between $F_{1}$ and $F_{0}$. Fant, Carlson, and Grandström (1974) had shown that the perceived quality of an unchanging formant pattern depended on $F_{0}$. Traunmüller supported his hypothesis in vowel identification experiments with listeners of Bavarian German, a dialect that has five distinct height categories. Perceived vowel height was well predicted on the basis of the distance, in Bark, between $F_{1}$ and $F_{0}$. This cue is a relational one: a given Bark separation of $F_{1}$ and $F_{0}$ is heard as the same vowel height, independent of the absolute frequency values of $F_{1}$ and $F_{0}$.

Traunmüller (1981) noted that his idea was reminiscent of an earlier hypothesis that vowel identity in general is specified by spatial patterns of excitation on the basilar membrane (Potter \& Steinberg, 1950; see also Chiba

This work was supported by research Grant 5 R01 DC 00427-07 from the National Institute on Deafness and Other Communication Disorders, National Institutes of Health, to the second author. The authors thank Terry Nearey for the idea for Experiment 1, and Renée Zakia, Geoff Amsel, Ann Syrdal, Hartmut Traunmüller, Neil Macmillan, and an anonymous reviewer for their comments on earlier drafts of this paper. Correspondence should be addressed to R. L. Diehl, Department of Psychology, 330 Mezes, University of Texas, Austin, TX 78712 (e-mail:diehl@psy.utexas.edu).
\& Kajiyama, 1941/1958). Formants are represented by a method known as place coding - the frequency of a formant is coded by the place of excitation along the basilar membrane. Following Zwicker and Feldtkeller (1967), Traunmüller noted that the Bark scale is approximately proportional to distance on the basilar membrane. Therefore the finding that $F_{1}-F_{0}$ Bark distance uniquely specifies vowel height is compatible with claiming that vowel height is specified by a fixed relation between $F_{1}$ and $F_{0}$ in the basilar membrane excitation pattern. ${ }^{2}$ In the excitation pattern, the "place" associated with $F_{0}$ is the position of the first harmonic (H1). Thus Traunmüller's results extended the earlier idea of Potter and Steinberg (1950) and Chiba and Kajiyama (1941/1958) to include an $F_{0}$-related feature (H1) as a critical part of the excitation pattern, in addition to formant frequencies. In fact, Traunmüller himself proposed a model of auditory pattern matching to account for his results, in which the Bark spacing of relevant parameters is derived from filtering operations analogous to those performed by the basilar membrane (pp. 1472-1473).

The idea of vowel height being specified by the relation between $F_{1}$ and $F_{0}$ in the basilar membrane excitation pattern has been quite influential in the vowel perception literature. Aside from Traunmüller (1981), other recent papers have used the $F_{1}-F_{0}$ Bark distance idea in models of speech perception (e.g., Bladon, Henton, \& Pickering, 1984; Hoemeke \& Diehl, 1994; Syrdal, 1985; Syrdal \& Gopal, 1986). In these papers, there is much to suggest that the authors intended to follow the lead of the earlier papers in using a place representation of frequency, with $F_{0}$ being represented by $\mathrm{H} 1$. Bladon et al. clearly indicated that their ideas for speaker normalization using $F_{1}-F_{0}$ distance were taken directly from the ideas of Potter and Steinberg (1950), and used a quotation from that earlier paper in which explicit reference is made to the basilar membrane (p. 62). Syrdal (1985) acknowledged that some of her ideas were quantitative versions of the 
Chiba and Kajiyama (1941/1958) and Potter and Steinberg (1950) papers, again making explicit that the distance hypothesis is based on spatial patterns in the peripheral auditory system (p. 129). Syrdal and Gopal's (1986) position is less clear. They began their paper with a reference to Chiba and Kajiyama (1941/1958) and Potter and Steinberg (1950) and the peripheral auditory system (p. 1086). However, in motivating the use of $F_{0}$ as a parameter functionally equivalent to a formant frequency, they cited Seneff's $(1984,1985)$ model of auditory processing. This model does not use place coding of frequency, but $F_{0}$ and formant frequencies are processed in the same manner (p. 1087). This suggests that Syrdal and Gopal had in mind a type of frequency coding distinct from the basilar membrane excitation pattern. Finally, Hoemeke and Diehl have also referred to Chiba and Kajiyama and Potter and Steinberg, again explicitly mentioning auditory distance in terms of the basilar membrane. One possible interpretation of these papers is that auditory distances are coded in the spatial pattern of excitation on the basilar membrane. ${ }^{3}$

In light of contemporary theories of pitch perception, the view of $F_{0}$ exerting influence by virtue of the frequency of H1 may appear antiquated. It has long been known from "missing fundamental" experiments that the pitch of a sound can be determined even when $\mathrm{H} 1$ is not physically present in the stimulus (Licklider, 1954; Schouten, 1938-1940; Seebeck, 1841, 1843; Small \& Campbell, 1961; Thurlow \& Small, 1955). In light of this, modern pitch perception theories do not postulate that energy at $\mathrm{H} 1$ is necessary for pitch to be perceived. For the present purposes, these theories may be divided into two classes.

Some theories attempt to retain place coding of frequency in spite of the missing fundamental experiments. For example, Terhardt's theory of virtual pitch (1974) suggested that in the absence of energy at $\mathrm{H} 1$, listeners infer the frequency of $\mathrm{H} 1$ from the frequencies of the harmonics that are actually represented in the energy spectrum. It is this inferred $\mathrm{H} 1$ that supports pitch perception. In this way, a place representation of frequency may be retained, since a tonotopic place is still associated with $F_{0}$ even in the absence of energy at that point.

However, others have completely abandoned the place representation, suggesting that pitch perception is driven by the periodicity caused by interference between two or more harmonics (e.g., Schouten, Ritsma, and Cardoza, 1962), or by cross-channel comparisons of auditory nerve firing patterns (Moore, 1973). The latter theories suggest that energy at $\mathrm{H} 1$ does not play a role in pitch perception at all.

The missing fundamental experiments appear to cause problems for excitation-pattern-based explanations of the role of $F_{1}-F_{0}$ distance in vowel height perception. However, this is not necessarily so. It is entirely possible that the use of $F_{0}$ in phonemic categorization and pitch perception is unrelated. In phonemic categorization, $F_{0}$ need not necessarily function in the same way that it does as a pitch cue. This is not to suggest that pitch perception in vowels differs from pitch perception in other sounds, but that the role of $F_{0}$ in phonemic categorization is different from its role in pitch perception. The perceived pitch of vowels might still conform to the principles derived from psychoacoustic experiments, but the perceived height might be more sensitive to the properties of the excitation pattern. Indeed, there is some evidence that place coding of $F_{0}$ is a better model of vowel height perception than of pitch perception. One of the arguments against the utility of place coding of $F_{0}$ in pitch perception is that below about $150 \mathrm{~Hz}$, changes in $F_{0}$ do not cause a change in the excitation pattern although pitch changes are still detectable. Place coding of $F_{0}$, therefore, cannot explain the differences between pitches below about $150 \mathrm{~Hz}$ (cf. Gulick, Gescheider, \& Frisina, 1989). Following suggestions by Traunmüller (1981), Di Benedetto (1994) found that at $F_{0}$ s below about $150 \mathrm{~Hz}$, vowel height judgments are not influenced by changes in $F_{0}$. This is what would be predicted if subjects were using a place-coded representation of $F_{0}$, because below this frequency the place code would remain the same regardless of the actual frequency of $F_{0}$. Hoemeke and Diehl (1994) found effects of $F_{0}$ on vowel height perception for $F_{0} \mathrm{~s}$ less than $150 \mathrm{~Hz}$. Lowering $F_{0}$ from $150 \mathrm{~Hz}$ to $125 \mathrm{~Hz}$ had a reliable effect on labeling of vowel continua. However, lowering $F_{0}$ further, to $125 \mathrm{~Hz}$, had no effect. This suggests that the lower limit of $F_{0}$ influence is somewhere between 125 and $150 \mathrm{~Hz}$-lower than that suggested by Di Benedetto (1994), but not so discrepant as to raise serious doubt about her results.

If the role of $F_{0}$ in vowel height perception derives from the representation of $\mathrm{H} 1$ in the basilar membrane excitation pattern, filtering $\mathrm{H} 1$ from vowels should disrupt classification of vowels by vowel height, despite the fact that pitch information is still available in the higher harmonics. One purpose of this study is to test that prediction. In Experiment 1, subjects classified a sevenmember $/ \mathrm{I} /-/ \varepsilon /$ continuum at five different levels of $F_{0}$. (The vowels $/ \mathrm{I} /$ and $/ \varepsilon /$ differ in height.) Hoemeke and Diehl (1994) found that under normal listening conditions, $F_{0}$ changes influenced vowel height judgments in such a way that higher $F_{0}$ s led to more $/ \mathrm{l} /$ responses, shifting the phoneme boundary toward the $/ \varepsilon /$ end of the continuum. In the present study, the stimuli were presented under three different conditions: unfiltered, filtered, filtered + noise. The unfiltered condition was a control: the stimuli were presented in unmodified form. In the filtered condition, $\mathrm{Hl}$ was removed in all of the series. However, $F_{0}$ was still encoded in the relationships among higher harmonics and in the overall periodicity of the sound, and so manipulations of $F_{0}$ would still affect the pitch of the sounds. In the filtered + noise condition, noise was added to the stimuli in the filtered condition to mask possible distortion products that might produce audible energy at the frequency of $\mathrm{H} 1$. One set of subjects performed the experiment at a presentation level of $70 \mathrm{~dB}$; a second group performed it at $50 \mathrm{~dB}$. The purpose of this lower presentation level group was to decrease further the likelihood of audible distortion products at the frequency of $\mathrm{H} 1$. 


\section{EXPERIMENT 1}

\section{Method}

Subjects. Subjects were introductory psychology students at the University of Texas at Austin, who participated in the experiment in partial fulfillment of a course requirement. All were native speakers of English and reported having normal hearing. All subjects performed the experiment in all three conditions, at either the $70-\mathrm{dB}$ or the $50-\mathrm{dB}$ presentation level.

Stimuli. Synthetic vowels ranging from $/ \mathrm{I} /$ to $/ \varepsilon /$ were synthesized by using the cascade-formant branch of the Klatt (1980) synthesizer implemented on a DEC VAXstation 3500. The endpoint formant frequencies for each set were equal to the mean adult male values of the first three formants reported by Peterson and Barney (1952) for the corresponding vowel categories. $F_{4}$ and $F_{5}$ were fixed at 3300 and $3850 \mathrm{~Hz}$. Five intermediate stimuli were synthesized by interpolating between the endpoint values of $F_{1}, F_{2}$, and $F_{3}$ in equal Bark steps. ${ }^{4}$ The formant frequencies in both $\mathrm{Hz}$ and Bark are presented in Table 1.

For each of the seven formant patterns, there were five constant $F_{0}$ values at equal frequency intervals ranging from 100 to $200 \mathrm{~Hz}$. Since the Bark scale is proportional to frequency $(100 \mathrm{~Hz}=1$ Bark) below $200 \mathrm{~Hz}$ (Traunmüller, 1990), $F_{0}$ varied in equal 0.25 Bark steps between 1 and 2 Bark.

The stimuli were $150 \mathrm{msec}$ in duration, the initial $15 \mathrm{msec}$ and final $10 \mathrm{msec}$ of which were multiplied by cosine-squared onset and offset functions. Each stimulus was normalized to a constant RMS amplitude as measured during the interval separating the rise and decay segments. Formant frequencies and $F_{0}$ were verified with the use of a spectrum analyzer.

For the filtered condition, stimuli were high-pass filtered with a "brick wall" digital filter at $225 \mathrm{~Hz}$. This removed energy at $\mathrm{H} 1$ for all five $F_{0}$ series, while for the $100-\mathrm{Hz} F_{0}$ series the filtering operation also removed the second harmonic. For the filtered + noise condition, white noise was low-pass filtered with a cutoff of $225 \mathrm{~Hz}$ and was added to each stimulus from the filtered condition. The spectrum level of the noise comfortably exceeded that of the most intense harmonic in the unfiltered stimulus set. For illustration, FFT spectra for the middle stimulus of the $150-\mathrm{Hz}$ continuum are shown in Figure 1, for each of the conditions.

Procedure. Separate groups of 24 subjects took part in each presentation level condition. Within each presentation level condition, the stimuli were blocked by filtering condition, and the order of presentation of the three filtering conditions was counterbalanced to yield six groups of 4 subjects each. Within each filtering condition, subjects identified 10 randomized blocks of the 35 stimuli ( 7 formant patterns $\times 5 F_{0}$ values). The subjects labeled the stimuli by pressing either of two response keys corresponding to the two vowel categories. They were given up to $2 \mathrm{sec}$ to respond, after which another $1 \mathrm{sec}$ elapsed before the next stimulus was presented. A short break was given between filtering conditions.

The stimuli, stored on an IBM AT computer, were output at a 10$\mathrm{kHz}$ sampling rate via a 16-bit $\mathrm{D} / \mathrm{A}$ converter, low-pass filtered at a $4.9-\mathrm{kHz}$ cutoff frequency, and presented to subjects binaurally

Table 1

Formant Values in Hertz and Bark for the Stimuli in Experiment 1

\begin{tabular}{|c|c|c|c|c|c|c|c|}
\hline & \multirow{2}{*}{$\begin{array}{c}\text { Formant } \\
\text { Pattern }\end{array}$} & \multicolumn{2}{|c|}{$F_{1}$} & \multicolumn{2}{|c|}{$F_{2}$} & \multicolumn{2}{|c|}{$F_{3}$} \\
\hline & & $\mathrm{Hz}$ & Bark & $\mathrm{Hz}$ & Bark & $\mathrm{Hz}$ & Bark \\
\hline \multirow[t]{6}{*}{$/ 1 /$} & 1 & 390 & 3.92 & 1990 & 12.98 & 2550 & 14.63 \\
\hline & 2 & 412 & 4.13 & 1964 & 12.89 & 2538 & 14.60 \\
\hline & 3 & 435 & 4.34 & 1939 & 12.80 & 2526 & 14.57 \\
\hline & 4 & 458 & 4.55 & 1914 & 12.71 & 2515 & 14.54 \\
\hline & 5 & 482 & 4.76 & 1889 & 12.63 & 2503 & 14.51 \\
\hline & 6 & 506 & 4.97 & 1864 & 12.54 & 2492 & 14.48 \\
\hline $\mid \varepsilon /$ & 7 & 530 & 5.18 & 1840 & 12.45 & 2480 & 14.45 \\
\hline
\end{tabular}
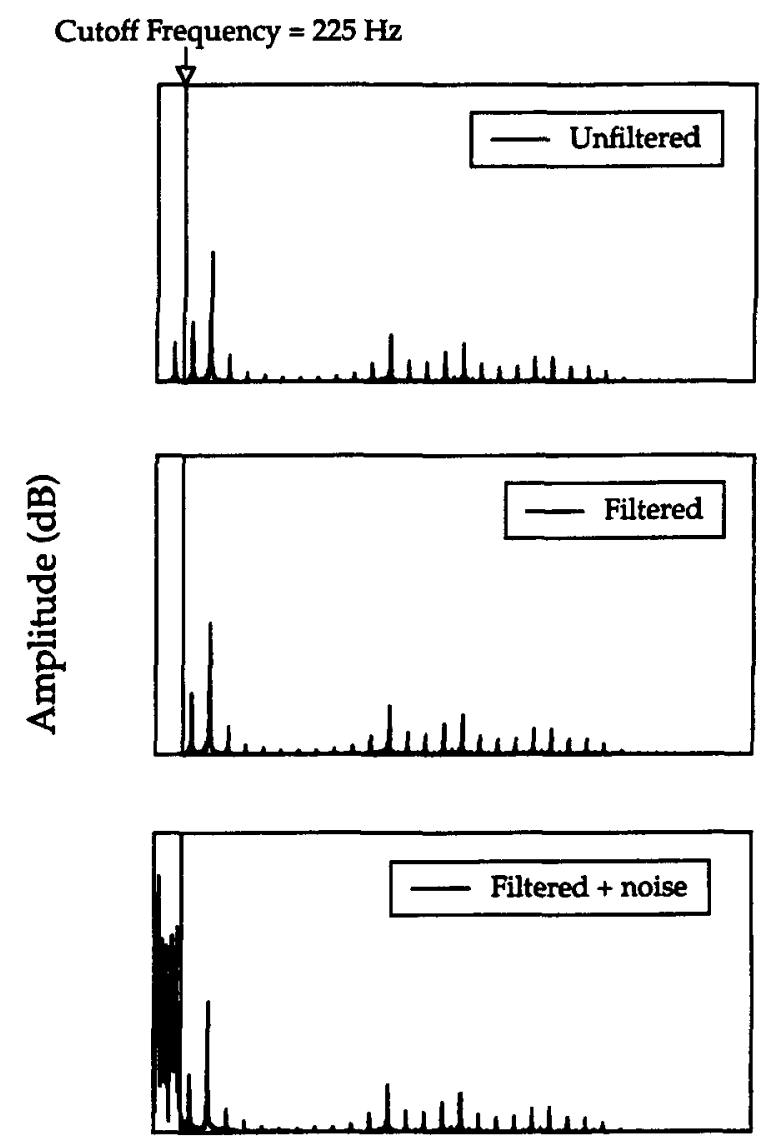

0

Frequency $(\mathrm{Hz})$

Figure 1. Spectra of a sample stimulus from each of the three filtering conditions. $F_{0}=150 \mathrm{~Hz}$.

over Beyer DT-100 earphones at 70 or $50 \mathrm{~dB}$ SPL. Up to 4 subjects, assigned to separate response stations in a double-walled soundattenuated chamber (Industrial Acoustics), served in each experimental session.

\section{Results and Discussion}

Identification functions collapsed across subjects are shown in Figure 2. Phoneme boundaries for the pooled identification functions were determined via linear regression on the $z$-transformed response probabilities. These boundaries are shown in Table 2. In all three filtering conditions, for both presentation levels, /I/ responses increased as $F_{0}$ increased; that is, the boundaries shifted toward the $/ \varepsilon /$ end of the continuum. Also shown in Table 2 are boundaries plotted as $F_{1}-F_{0}$ Bark distances. Boundaries were generally very close to the 3-3.5 Bark range predicted by Syrdal and Gopal (1986), and for $F_{0}$ s up to $175 \mathrm{~Hz}$, they decreased as a function of increasing $F_{0}$. This reflects the fact that $F_{1}$ boundary shifts (in Bark) were smaller than the $F_{0}$ steps (in Bark) that produced them. Such an effect, also observed by Hoemeke and Diehl (1994), is compatible with production data reported by Syrdal and Steele (1985) indicating 


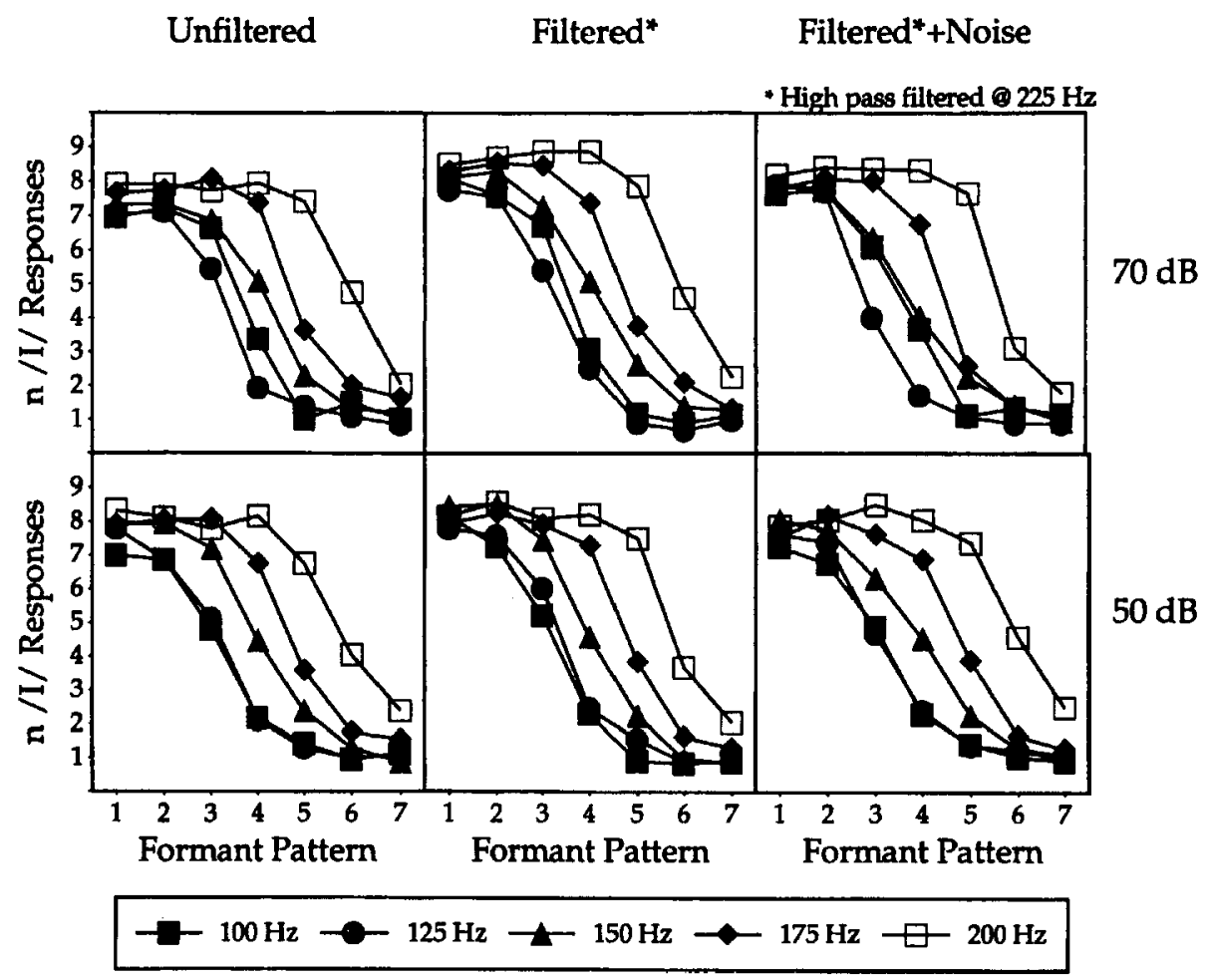

Figure 2. Identification functions combined across subjects, Experiment 1.

that $F_{1}$ increases by only small amounts in proportion to increasing $F_{0}$. These perceptual and production results suggest that $F_{1}-F_{0}$ distance is not an invariant correlate of vowel height, although Syrdal and Steele showed that vowels could still be divided into appropriate phonetic categories by positing boundaries along the $F_{1}-F_{0}$ distance dimension.

The raw identification scores were entered into an analysis of variance (ANOVA), with two between-subjects variables (presentation level, counterbalancing order) and three within-subjects variables (filtering condition, formant pattern, and $F_{0}$ ). The main effects of presentation level and counterbalancing order were not significant $[F(1,41)=0.27, p=.61 ; F(5,41)=0.40, p>.05$, respectively]. There were highly significant main effects of formant pattern $[F(6,246)=143.63, p \leq .0001]$ and
$F_{0}[F(4,164)=110.30, p \leq .0001]$. Newman-Keuls comparisons between the means for each formant pattern showed that all comparisons were significant $(p \leq .01)$ except for the comparisons between Formant Patterns 1 and 2 and between Formant Patterns 6 and $7(p>.05)$. All comparisons between $F_{0}$ condition means were significant ( $p \leq .01$ ), except for the comparison between the $100-$ and $125-\mathrm{Hz} F_{0}$ conditions $(p>.05)$. There was a highly significant interaction between formant pattern and $F_{0}[F(24,948)=51.61, p \leq .0001]$. Analyses of simple effects revealed that the effect of formant pattern was highly significant at every level of $F_{0}(p \leq .001)$. Although the effect of $F_{0}$ was highly reliable at every level of formant pattern $(p \leq .01)$, the effect, as indicated by the magnitude of the $F$ ratios, was most reliable for the formant patterns near the middle of the continuum. Hoe-

Table 2

Phoneme Boundaries for Experiment 1

\begin{tabular}{llllll}
\hline \multicolumn{5}{c}{$F_{0}$} \\
& \multicolumn{5}{c}{} \\
\cline { 2 - 6 } & $100 \mathrm{~Hz}$ & $125 \mathrm{~Hz}$ & $150 \mathrm{~Hz}$ & $175 \mathrm{~Hz}$ & $200 \mathrm{~Hz}$ \\
\hline $70 \mathrm{~dB}$ & & & & & \\
$\quad$ Unfiltered & $3.0(3.4)$ & $2.7(3.0)$ & $3.5(2.9)$ & $4.3(2.9)$ & $5.8(2.9)$ \\
$\quad$ Filtered* & $3.3(3.4)$ & $2.9(3.1)$ & $3.9(3.0)$ & $4.5(2.9)$ & $6.2(3.0)$ \\
$\quad$ Filtered + noise* & $3.2(3.4)$ & $2.8(3.0)$ & $3.5(2.9)$ & $4.0(2.8)$ & $5.5(2.9)$ \\
$50 \mathrm{~dB}^{\text {Unfiltered }}$ & & & & & \\
$\quad$ Filtered* & $2.6(3.3)$ & $2.8(3.1)$ & $3.6(3.0)$ & $4.3(2.9)$ & $5.6(2.9)$ \\
$\quad$ Filtered + noise* & $2.9(3.3)$ & $3.1(3.1)$ & $3.8(3.0)$ & $4.3(2.9)$ & $5.6(2.9)$ \\
\hline
\end{tabular}

Note-Boundaries are measured in stimulus step, with $F_{1}-F_{0}$ Bark distances in parentheses. *High-pass cutoff frequency $=225 \mathrm{~Hz}$. 
meke and Diehl (1994) found similar main effects and interactions involving formant pattern and $F_{0}$ for an $/ \mathrm{I} /-\mathbf{\varepsilon} /$ continuum.

Most important for this study is whether there were any differences between filtering conditions. There was a significant main effect of filtering condition $[F(2,82)=$ $4.86, p \leq .01]$. Pairwise comparisons between the means showed that there were slightly more /I/ responses ( $p \leq$ $.05)$ in the filtered condition than in the unfiltered and filtered + noise conditions (mean number of $/ \mathrm{I} /$ responses of 4.97 vs. 4.75 and 4.72 , respectively). This slight difference is reflected in the fact that the mean phoneme boundaries (Table 2 ) are generally shifted toward the $/ \varepsilon /$ end of the continuum for the filtered condition, relative to the unfiltered and filtered + noise conditions. This difference is surprising: if any differences were to be expected across filtering conditions, one would expect either the unfiltered condition to be different from the two filtered conditions, or the filtered + noise condition to be different from the unfiltered and filtered conditions (if filtering alone had not removed all energy at $F_{0}$ ).

The cause of the main effect of filtering condition may be traced via the significant interactions. Analysis of simple effects showed that the significant interaction between filtering condition and counterbalancing order $[F(10,82)=3.09, p \leq .01)$ was due to the effect of filtering condition being significant $(p \leq .001)$ for only one of the counterbalancing order groups-namely, the group of subjects hearing the order "unfiltered, filtered + noise, filtered" (Group 2). Upon inspection of the individual data, the large effect of filtering condition in Group 2 appears to be due to the fact that 4 of the 6 subjects who demonstrated large differences in response probabilities across filtering conditions were in Group 2. All tended to make fewer $/ \mathrm{l} /$ responses in either the unfiltered or the filtered + noise conditions, or both, than in the filtered condition. The other 2 subjects showing differences across filtering conditions also follow this pattern but were in different counterbalancing order groups.

The response patterns of the aforementioned 6 subjects differed from those of the rest of the subjects for the formant patterns for which there was a large number of /I/ responses. Consistent with this, the interaction between filtering condition and formant pattern was also significant $[F(12,492)=2.34, p \leq .01]$, with analysis of simple effects showing that the effects of filtering condition were reliable only for Formant Pattern 1 and Formant Pattern $2(p \leq .05)$ and Formant Pattern $3(p \leq .01)$.

If one combines the effects of the two significant interactions described in the paragraphs above, the significant interaction between filtering condition, formant pattern, and counterbalancing order $[F(60,492)=1.87$, $p \leq .001$ ) can be attributed to the fact that the interaction between formant pattern and filtering condition was significant only in Group $2(p \leq .0001)$, the counterbalancing order group in which there was a significant effect of filtering condition. Thus the interactions showed that the main effect of filtering condition was localized to a small number of subjects, and also only to formant patterns near the $/ \mathrm{I} /$ end of the continuum. This latter result might be expected, since the /1/-like stimuli have the lowest $F_{1} \mathrm{~s}$ and so are the most likely to be affected by the removal of low frequency harmonics.

Importantly, the interaction between filtering condition and $F_{0}$ was not significant $[F(8,328)=1.05, p>$ $.05]$. This means that the effect of $F_{0}$ did not differ across filtering conditions. However, the interaction between filtering condition, formant pattern, and $F_{0}[F(48,240)=$ $1.56, p \leq .01]$ was significant. The cause of this appears to be that in the filtered + noise condition, the effect of $F_{0}$ at Formant Pattern 1 was not significant $(p>.05)$, whereas in all other cases the effects of $F_{0}$ and formant pattern on each other were highly significant $(p \leq .001)$. All other effects in the ANOVA were nonsignificant $(p>.05)$.

Overall, the results of Experiment 1 demonstrated a highly significant effect of $F_{0}$ on vowel height perception, even when there is no energy in the signal at $\mathrm{H} 1$. The magnitude of the effect did not differ across filtering conditions. It appears that the effect of $F_{0}$ on vowel height perception does not derive from place coding of $\mathrm{Hl}$ in the excitation pattern. However, before this conclusion is accepted, one other possibility must be considered. Removing the energy at $\mathrm{H} 1$ also narrows the excitation pattern between $F_{1}$ and the lowest frequency represented in the excitation pattern. With $\mathrm{H} 1$ removed, the lowest frequency is the second harmonic (H2). Di Benedetto (1994) suggested that $F_{0}$ influences vowel height perception because $\mathrm{H} 1$ may serve as a low frequency "anchor point" in the excitation pattern, in the context of which $F_{1}$ is interpreted. At low $F_{0} \mathrm{~s}(<150 \mathrm{~Hz})$, excitation due to $\mathrm{Hl}$ is at the apical end of the basilar membrane, so the anchor point coincides with the extreme end of the excitation pattern. Up to $150 \mathrm{~Hz}$, changes in $F_{0}$ do not alter the excitation pattern. The anchor point remains coincident with the end of the scale, and vowel height judgments are not affected. As $F_{0}$ increases above $150 \mathrm{~Hz}, F_{0}$-related excitation is no longer coincident with the apical end of the basilar membrane. Changes in $F_{0}$ do alter the position of the anchor point, and so vowel height judgments are influenced by $F_{0}$. Consistent with these hypotheses, Di Benedetto's experiments showed that changes in $F_{0}$ below about $150 \mathrm{~Hz}$ did not influence vowel height perception, but rather perceived vowel height was well predicted by $F_{1}$ alone. As $F_{0}$ was increased above $150 \mathrm{~Hz}$, perceived vowel height was increasingly influenced by $F_{0}$, with $F_{1}-F_{0}$ distance being a better predictor of vowel height than was $F_{1}$ alone.

The anchor point hypothesis could explain the persistence of the $F_{0}$ effect in Experiment 1 without abandoning the spatial pattern representation. When $\mathrm{Hl}$ is removed, the second harmonic becomes the anchor point, since it is the lowest frequency in the excitation pattern. Because the frequency of the second harmonic is perfectly correlated with $F_{0}$, increasing $F_{0}$ would have qualitatively similar effects on the position of the anchor point regardless of whether or not $\mathrm{H} 1$ was physically present. ${ }^{5}$

However, the anchor hypothesis does not predict certain aspects of the existing data. Although the anchor hy- 
pothesis predicts qualitatively similar results across filtering conditions, it predicts quantitative differences between unfiltered and filtered conditions, differences that were not wholly borne out in Experiment 1 . First, the difference between the second harmonics of adjacent $F_{0}$ series was $50 \mathrm{~Hz}$, as opposed to a $25-\mathrm{Hz}$ difference in $F_{0}$. If the role of $F_{0}$ is simply that of an anchor point, the larger between-series step size in anchor frequency should have resulted in larger between-series effects for the filtered conditions than for the unfiltered condition. This was not the case. Inspection of the phoneme boundaries (Table 2) gives no indication of a larger shift in the filtered or filtered + noise conditions than in the unfiltered condition.

Second, in the filtered conditions, the distance between the anchor point and $F_{1}$ was uniformly smaller than in the unfiltered condition. If this distance is a cue for vowel height, the stimuli in the filtered conditions should have appeared uniformly more $/ \mathrm{I} /$-like than those in the unfiltered condition. The significant main effect of filtering condition in Experiment 1 provides some evidence for this: there were more $/ \mathrm{I} /$ responses in the filtered condition than in the other two conditions (although the main effect was small, and it seemed to be limited to a few subjects and formant patterns). The phoneme boundaries (Table 2) were also shifted toward the $/ \varepsilon /$ end of the continuum for the filtered condition, relative to the other two conditions. This is a difference in the expected direction relative to the unfiltered condition, if listeners were using $\mathrm{H} 2$ as an anchor rather than $\mathrm{Hl}$. However, since filtering changed the frequency of the anchor point by at least $100 \mathrm{~Hz}$, and the stimulus step size is never greater than $30 \mathrm{~Hz}$, phoneme boundaries in the filtered conditions would have had to be at least three stimulus steps away from the boundaries in the unfiltered condition in order to offset the effects of using $\mathrm{H} 2$ as an anchor instead of $\mathrm{H} 1$. Inspection of the phoneme boundaries (Table 2) reveals that this was not the case. The size of the boundary shifts was much too small to provide support for the anchor hypothesis.

Further, the anchor hypothesis predicts that the boundaries should have shifted toward $/ \varepsilon /$ for the filtered + noise condition as well as the filtered condition, which was not the case. Giving the anchor hypothesis the benefit of the doubt, it is conceivable that listeners interpreted the noise as having masked the anchor point and so inferred that it was somewhere in the noise. The inferred anchor points would have been at lower frequencies than those used in the filtered condition (since the noise covered a frequency region that was lower than any of the second harmonics), and so boundaries would have been more in line with the unfiltered condition. But then it is difficult to explain why there was a systematic effect of $F_{0}$ if the position of the anchor point was masked and was, presumably, uncertain. It could also be argued in favor of the anchor hypothesis that the frequency of $\mathrm{Hl}$ was inferred from the audible spectrum of the sounds, and that this was then used as the anchor point. However, this undermines the original hypothesis that listeners were using properties of the excitation pattern to judge vowel height.
Although the results of Experiment 1 do not support the anchor hypothesis, the test was not direct. Experiment 2 was conceived as a direct test. Stimuli from Experiment 1 were configured so that the anchor hypothesis predicts an effect of $F_{0}$ in the opposite direction to that found in Experiment 1 . The $150-\mathrm{Hz}, 175-\mathrm{Hz}$, and $200-\mathrm{Hz} F_{0}$ stimuli from Experiment 1 were presented for identification in both an unfiltered and a filtered condition. These three $F_{0}$ conditions were chosen because the effects of $F_{0}$ are largest in this range (Di Benedetto, 1994; Hoemeke \& Diehl, 1994). In the filtered condition, stimuli were low-pass filtered in such a way that the frequency of the lowest harmonic present in the stimuli varied inversely, rather than directly, with $F_{0}$. If the influence of $F_{0}$ derives from the role of $\mathrm{Hl}$ as an anchor point in the excitation pattern, we should expect more/// responses as $F_{0}$ decreases, since decreasing $F_{0}$ would decrease the distance between $F_{1}$ and the anchor point. If the role of $F_{0}$ derives from its role qua $F_{0}$, then we should expect more / $\mathrm{I} /$ responses as $F_{0}$ increases, as in Experiment 1 . A filtered + noise condition was not used, owing to the possible confounding effects of the noise, which were discussed above. Because Experiment 1 revealed no effects of presentation level, stimuli were presented only at $50 \mathrm{~dB}$.

\section{EXPERIMENT 2}

\section{Method}

Subjects. Subjects were introductory psychology students at the University of Texas at Austin, who participated in partial fulfillment of a course requirement. All were native speakers of English and reported having normal hearing. None had taken part in Experiment 1 .

Stimuli. The stimuli were based on the $150-\mathrm{Hz}, 175-\mathrm{Hz}$, and $200-\mathrm{Hz} F_{0}$ stimuli from Experiment 1 . For the unfiltered condition, the stimuli were not altered in any way. For the filtered condition, the $150-\mathrm{Hz} F_{0}$ stimuli were high-pass filtered at $375 \mathrm{~Hz}$, and the $175-\mathrm{Hz} F_{0}$ stimuli were filtered at $265 \mathrm{~Hz}$. This removed $\mathrm{H} 1$ and $\mathrm{H} 2$ of the $150-\mathrm{Hz} F_{0}$ stimuli, and $\mathrm{H} 1$ of the $175-\mathrm{Hz} F_{0}$ stimuli. The $200-\mathrm{Hz} F_{0}$ stimuli were left unchanged. Thus the lowest frequencies present in the $150-\mathrm{Hz}, 175-\mathrm{Hz}$, and $200-\mathrm{Hz} F_{0}$ continua were 450,350 , and $200 \mathrm{~Hz}$, respectively, varying inversely with $F_{0}$.

Procedure. Seventeen subjects identified the stimuli in both filtering conditions. The stimuli were blocked by filtering condition, and the order of presentation of these conditions was counterbalanced. Within each filtering condition, subjects identified 10 randomized blocks of the 35 stimuli ( 7 formant patterns $\times 5 F_{0}$ values). Subjects labeled the stimuli by pressing either of two response keys corresponding to the two vowel categories. They were given up to $2 \mathrm{sec}$ to respond, after which another $1 \mathrm{sec}$ elapsed before the next stimulus was presented. A short break was given between filtering conditions.

Stimulus storage and presentation parameters were as described for Experiment 1, except that only the 50-dB SPL presentation level was used. Again, up to 4 subjects at separate response stations in the sound-attenuated chamber served in each experimental session.

\section{Results and Discussion}

Identification functions collapsed across subjects are shown in Figure 3. Phoneme boundaries for the pooled identification functions are shown in Table 3. As in Ex- 
periment 1 , there was a large effect of $F_{0}$. In both filtered and unfiltered conditions, phoneme boundaries moved toward the $/ \varepsilon /$ end of the continuum as $F_{0}$ was increased. Phoneme boundaries as a whole were shifted toward the /I/ end of the continuum in the filtered condition, especially for the $150-\mathrm{Hz} F_{0}$ series (recall that it was the $150-\mathrm{Hz} F_{0}$ series that was most drastically filtered). Traunmüller (personal communication, February 20,1994) found a similar qualitative shift in an unpublished experiment and explained the result by proposing that the removal of lower partials - especially those near the formant peakcan cause the "effective" $F_{1}$ to be shifted upward in frequency. This would have the effect of making all the vowels sound more $/ \varepsilon /$-like and so would shift all the boundaries toward the $/ \mathrm{I} /$ end of the continuum. This phenomenon is also likely to be responsible for the $F_{1}-F_{0}$ distance boundary being smallest for the $150-\mathrm{Hz} F_{0}$ series in the filtered condition, contrary to all other conditions where (if anything) $F_{1}-F_{0}$ distance decreased as a function of increasing $F_{0}$. If effective $F_{1}$ is increased while nominal $F_{1}$ stays the same, $F_{1}-F_{0}$ distance at the boundary will necessarily decrease, since listeners need a smaller nominal $F_{1}-F_{0}$ distance in order to achieve the same effective $F_{1}-F_{0}$ distance.

The direction of the shift within the filtered condition is opposite to that which would be predicted if the lowest harmonics physically present were being used as anchors. The raw identification scores were entered into an ANOVA, with one between-subjects variable (counterbalancing order) and three within-subjects variables (filtering condition, formant pattern, and $F_{0}$ ). The main effects of presentation order and filtering condition were not significant $[F(1,15)=0.173, p>.05, F(1,15)=3.01$, $p>.05$, respectively]. There were significant main effects of formant pattern $[F(6,90)=25.78, p \leq .0001]$ and $F_{0}[F(2,30)=16.35, p \leq .0001]$. Newman-Keuls tests showed that of the comparisons between $F_{0}$ levels, those between the $150-\mathrm{Hz}$ and $175-\mathrm{Hz}$, and between the
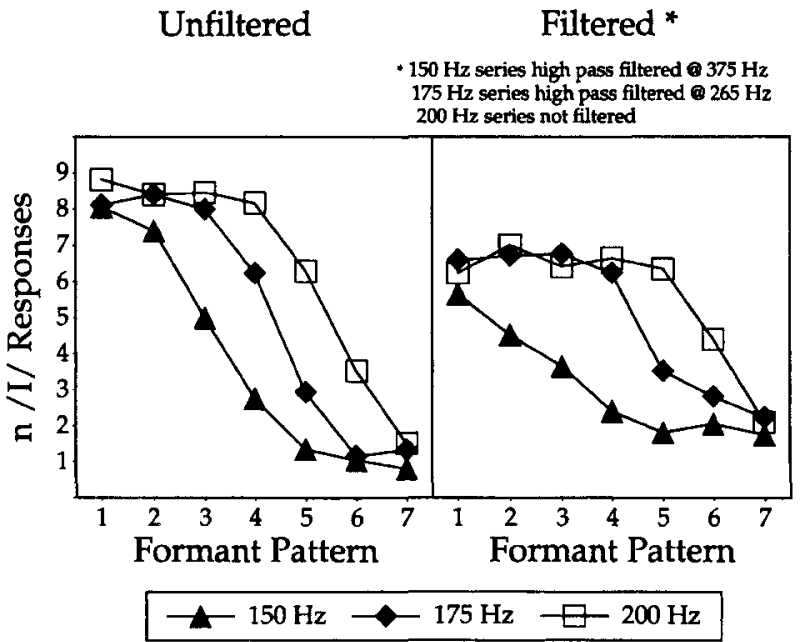

Figure 3. Identification functions combined across subjects, Experiment 2.
Table 3

Phoneme Boundaries for Experiment 2

\begin{tabular}{llll}
\hline & \multicolumn{3}{c}{$F_{0}$} \\
\cline { 2 - 4 } $50 \mathrm{~dB}$ & $150 \mathrm{~Hz}$ & $175 \mathrm{~Hz}$ & $200 \mathrm{~Hz}$ \\
\hline Unfiltered & $3.0(2.9)$ & $4.1(2.8)$ & $5.2(2.8)$ \\
Filtered & $1.2(2.5)^{*}$ & $3.9(2.8) \dagger$ & $4.9(2.7) \ddagger$ \\
\hline
\end{tabular}

Note - Boundaries are measured in stimulus step, with $F_{1}-F_{0}$ Bark distances in parentheses. *High-pass cutoff frequency $=375 \mathrm{~Hz}$. †Highpass cutoff frequency $=265 \mathrm{~Hz} . \quad \$$ Not filtered.

$150-\mathrm{Hz}$ and $200-\mathrm{Hz}, F_{0}$ conditions were significant at $p \leq .01$, and the comparison between the $175-\mathrm{Hz}$ and $200-\mathrm{Hz} F_{0}$ conditions was significant at $p \leq .05$. Comparisons between formant pattern levels were significant at $p \leq .01$, except for those between Formant Patterns 1 and 4 , and between Formant Patterns 2 and $4(p \leq .05)$, and those between Formant Patterns 1 and 2, Formant Patterns 1 and 3, Formant Patterns 2 and 3, Formant Patterns 3 and 4, Formant Patterns 5 and 6, and Formant Patterns 6 and $7(p>.05)$. There was a significant interaction between $F_{0}$ and formant pattern $[F(12,180)=$ $9.04, p \leq .0001]$. Analysis of simple effects showed that this was due to a greater reliability of $F_{0}$ effects near the middle of the continua than at the endpoints. However, all simple effects were significant $(p \leq .01)$, except for the effect of $F_{0}$ at Formant Pattern $1(p=.49)$, the effect of $F_{0}$ at Formant Pattern $2(p=.02)$, and the effect of $F_{0}$ at Formant Pattern $7(p=.192)$. The only other significant effect in the ANOVA was the interaction between filtering condition and formant pattern $[F(6,90)=6.84$, $p \leq .0001]$. Analyses of simple effects determined that this interaction was due to there being a more reliable effect of filtering condition on formant patterns at the /I/ end of the continuum than at the $/ \varepsilon /$ end of the continuum: $p \leq .01$ for Formant Pattern 1 and Formant Pattern 2, $p \leq .05$ for Formant Pattern 3, and $p>.05$ for the rest of the formant patterns. This interaction between filtering condition and formant pattern is not surprising, since the formant patterns that differed between filtering conditions were those with the lowest $F_{1} \mathrm{~s}$. Filtering would have interfered with the $F_{1}$ region of these stimuli more than for those with higher $F_{1} \mathrm{~s}$.

As in Experiment 1, the interaction between $F_{0}$ and filtering condition was not significant $[F(2,30)=0.68$, $p=.52]$. We can therefore conclude that Experiment 2 provides no support for the anchor hypothesis. The effect of $F_{0}$ on vowel height perception does not derive from its role as an anchor point in the excitation pattern.

\section{GENERAL DISCUSSION}

The experiments reported in this paper have shown that the effect of $F_{0}$ on vowel height perception is very robust, at least for the $/ \mathrm{I} /-/ \varepsilon /$ distinction. In both experiments, the effect of increasing $F_{0}$ was to increase the number of $/ \mathrm{I} /$ responses, shifting the boundary toward the $/ \varepsilon /$ end of the continuum. This effect persisted under various conditions of filtering out the lowest harmonics. 
It is therefore very clear that the role of $F_{0}$ in vowel height perception does not derive simply from coding of $\mathrm{Hl}$ in the basilar membrane excitation pattern. Experiment 2 shows that the influence of $F_{0}$ is not due to the role of $\mathrm{Hl}$ as a low frequency anchor in the excitation pattern. Rather, the persistence of $F_{0}$ effects in the absence of $\mathrm{Hl}$ suggests that the same representation of $F_{0}$ is used in both pitch and vowel height judgments.

This role of $F_{0}$ in vowel height judgments may be made to conform to current pitch perception theories in one of two ways. Traunmüller (personal communication, February 20,1994 ) suggested that a place representation of $F_{0}$ could be retained in vowel height perception if a mechanism such as that proposed by Terhardt (1974) for pitch perception were involved. The listener could infer the position of $\mathrm{Hl}$ as pitch is computed from higher harmonics. This is plausible, and it would explain the results of our study. However, it must be noted that Terhardt's theory of pitch perception is not universally accepted, and so whether such a mechanism actually exists is a matter of debate.

Many contemporary theories of pitch perception have abandoned place coding of frequency, relying instead on periodicity in the waveform. Compatible with this is Delgutte and Kiang's (1984) suggestion that vowel spectra could also be represented in the discharge patterns of auditory nerves. Formants would be indicated by a high degree of synchrony with the formant frequency across auditory nerves with a range of characteristic frequencies (CFs). $F_{0}$ information would be present in synchrony with the fundamental in fibers with CFs close to $F_{0}$, and also in higher CF fibers. These high CF fibers would be responding to more than one harmonic, and interactions between the harmonics would give the driving signal a periodicity with a frequency equal to $F_{0}$. In the absence of $\mathrm{H} 1, F_{0}$ information would presumably be contained almost exclusively in these higher CF fibers. Syrdal (personal communication, February 20, 1994) has suggested to us that auditory distance theories are not necessarily dependent on a place representation of frequency and has pointed out Syrdal and Gopal's (1986) allusion to Seneff's $(1984,1985)$ synchrony-based auditory model as evidence for this.

A final issue concerns the source of the inverse correlation between $F_{1}$ and $F_{0}$ across vowels of varying height. Several theorists have attributed this correlation to some form of anatomical coupling between the tongue body and larynx such that a higher $F_{0}$ is a passive physical consequence of a higher tongue position (Honda, 1983; Ladefoged, 1964; Ohala \& Eukel, 1987). A natural extension of this view is that the cue value of $F_{0}$ with respect to vowel height derives from the listener's having experienced the natural inverse correlation between $F_{1}$ and $F_{0}$. A recent study by Whalen, Levitt, Hsiao, and Smorodinsky (1995) claims to support the hypothesis that $F_{0}$ variation is a passive consequence of differences in tongue height. A single listener transcribed the infant babbling of 6-, 9-, and 12-month-olds, and the $F_{0}$ of the vowels was also measured. On the average, vowels tran- scribed as [+high] had higher $F_{0} \mathrm{~s}$. Whalen et al. argued that such variation of $F_{0}$ is unlikely to be the result of active control of $F_{0}$ to enhance vowel distinctiveness. However, because the formants of infant speech are quite indistinct, it is possible that the single listener relied mainly on the $F_{0}$ cue for vowel height in such a way that vowels with higher $F_{0}$ s tended to be transcribed as [+high] irrespectively of actual height.

An alternative to the hypothesis that $F_{0}$ variation is a passive consequence of tongue height differences is that talkers actively control $F_{0}$, or $F_{1}-F_{0}$ distance, in order to enhance vowel height distinctions (Hoemeke \& Diehl, 1994). Syrdal and Steele (1985) found that heightrelated $F_{0}$ differences were greater for vowels in stressed position than in unstressed position. This is compatible with the hypothesis of active pitch regulation, since one would expect talkers to produce larger $F_{0}$ differences in the syllables that carry greater communicative load. Further, in several electromyographic studies of adult talkers of various languages (Auteserre, Di Cristo, \& Hirst, 1986; Dyhr, 1990; Honda \& Fujimura, 1991; Vilkman, Aaltonen, Raimo, Arajävi, \&Oksanen, 1989), cricothyroid activity was found to be positively correlated with tongue height. Because the cricothyroid is the primary muscle implicated in the active regulation of pitch, these results argue against an explanation of height-related $F_{0}$ variation based on passive physical factors alone. The claim that $F_{0}$ is controlled by (adult) talkers to enhance the auditory distinctiveness of height contrasts is at least plausible in view of the robust perceptual effect of $F_{0}$ observed in the present study.

\section{REFERENCES}

Auteserre, D., Di Cristo, A., \& Hirst, D. J. (1986). Approche physiologique des intonations de base du français: Cricothroidien et fréquence fondamentale [Physiological approach to French intonation: The cricothyroid and fundamental frequency]. In Proceedings of the 15th Journée d'Étude de la Parole (Aix-en-Provence) (pp. 3741). Aix-en-Provence.

Bladon, R. A. W., Henton, C. G., \& Pickering, J. B. (1984). Towards an auditory theory of speaker normalization. Language \& Communication, 4, 59-69.

CHIBA, T., \& KaJIYAMA, M. (1958). The vowel: Its nature and structure. Tokyo: Phonetic Society of Japan. (Original edition published 1941)

Delattre, P., Liberman, A. M., Cooper, F. S., \& Gerstman, L. J. (1952). An experimental study of the acoustic determinants of vowel color: Observations on one- and two-formant vowels synthesized from spectrographic patterns. Word, 8, 195-210.

Delgutte, B., \& Kiang, N. Y. S. (1984). Speech coding in the auditory nerve: I. Vowel-like sounds. Journal of the Acoustical Society of America, 75, 866-878.

Di BenedetTo, M.-G. (1994). Acoustic and perceptual evidence of a complex relation between $F_{1}$ and $F_{0}$ in determining vowel height. Journal of Phonetics, 22, 205-224.

DyHR, N. (1990). The activity of the cricothyroid muscle and the intrinsic fundamental frequency in Danish vowels. Phonetica, 47, 141-154.

Fant, G., Carlson, R., \& GRANDSTRÖM, B. (1974). The [e]-[o] ambiguity. Proceedings of the Speech Communication Seminar, Stockholm, 3, 117-121.

Gulick, W. L., Gescheider, G. A., \& Frisina, R. D. (1989). Hearing. New York: Oxford University Press.

Hoemeke, K. A., \& Diehl, R. L. (1994). Perception of vowel height: The role of $F_{1}-F_{0}$ distance. Journal of the Acoustical Society of America, 96, 661-674. 
HONDA, K. (1983). Relationship between pitch control and vowel articulation. In D. M. Bless \& J. H. Abbs (Eds.), Vocal fold physiology: Contemporary research and clinical issues (pp. 286-297). San Diego, CA: College Hill Press.

HoNDA, K., \& Fujimura, O. (1991). Intrinsic vowel $F_{0}$ and phrasefinal $F_{0}$ lowering: Phonological versus biological explanations. In J. Gauffin \& B. Hammarberg (Eds.), Vocal fold physiology: Acoustic, perceptual, \& physiological aspects of voice mechanisms (pp. 149-157). San Diego, CA: Singular.

House, A. S., \& Fairbanks, G. (1953). The influence of consonant environment upon the secondary acoustical characteristics of vowels. Journal of the Acoustical Society of America, 25, 105-113.

KLATT, D. H. (1980). Software for a cascade/parallel formant synthesizer. Journal of the Acoustical Society of America, 67, 971-995.

LADEFOGED, P. (1964). A phonetic study of West African languages (West African Language Monograph Series I). Cambridge: Cambridge University Press.

Lehiste, 1., \& Peterson, G. E. (1961). Some basic considerations in the analysis of intonation. Journal of the Acoustical Society of America, 33, 419-425.

LICKLIDER, J. C. R. (1954). Periodicity pitch and place pitch [Abst ract]. Journal of the Acoustical Society of America, 26, 945.

Miller, R. L. (1953). Auditory tests with synthetic vowels. Journal of the Acoustical Society of America, 25, 114-121.

MoORE, B. C. J. (1973). Some experiments relating to the perception of complex tones. Quarterly Journal of Experimental Psychology, 25, 451-475.

Ohala, J. J., \& EUKel, B. M. (1987). Explaining the intrinsic pitch of vowels. In R. Channon \& L. Schockey (Eds.), In honor of Ilse Lehiste: Ilse Lehiste Pühenduestos (pp. 207-215). Dordrecht: Foris.

Peterson, G. E., \& Barney, H. (1952). Control methods used in a study of the vowels. Journal of the Acoustical Society of America, 24, 175-184.

Potter, R. K., Kopp, G., \& Green, H. (1947). Visible speech. New York: Van Nostrand Reinhold.

POTTER, R. K., \& STEINBERG, J. C. (1950). Toward the specification of speech. Journal of the Acoustical Society of America, 22, 807-820.

Schouten, J. F. (1938-1940). Five articles on the perception of sound. Eindhoven: Institute for Perception.

Schouten, J. F., Ritsma, R. J., \& Cardoza, B. L. (1962). Pitch of the residue. Journal of the Acoustical Society of America, 34, 14181424.

SEEBECK, A. (1841). Beobachtungen über einige Bedingungen der Entstehung von Tönen [Observations on a condition for the origin of tones]. Annalen der Physick und Chemie, 53, 4l 7-436.

SEEBECK, A. (1843). Uber die Sirene [On the siren]. Annalen der Physick und Chemie, 60, 449-481.

SENEFF, S. (1984). Pitch and spectral estimation of speech based on an auditory synchrony model. In Speech Communication Group Working Papers (Vol. 4, pp. 43-56). Cambridge, MA: MIT, Research Laboratory of Electronics.

SENEFF, S. (1985). Pitch and spectral analysis of speech based on an auditory synchrony model. Unpublished doctoral dissertation, Massachusetts Institute of Technology.

SMALL, A. M., \& CAMPBell, R. A. (1961). Masking of pulsed tones by bands of noise. Journal of the Acoustical Society of America, 33, $1570-1576$.

SYRDAL, A. K. (1985). Aspects of a model of the auditory representation of American English vowels. Speech Communication, 4, 121-135.

SYRDAL, A. K., \& Gopal, H. S. (1986). A perceptual model of vowel recognition based on the auditory representation of American English vowels. Journal of the Acoustical Society of America, 79, 1086-1100.

SYRDAl, A. K., \& STEELE, S. A. (1985). Vowel $F_{1}$ as a function of fundamental frequency [Abstract]. Journal of the Acoustical Society of America, 78, S56.
TERHARDT, E. (1974). Pitch, consonance, \& harmony. Journal of the Acoustical Society of America, 55, 1061-1069.

Thurlow, W. R., \& SMall, A. M. (1955). Pitch perception for certain periodic auditory stimuli. Journal of the Acoustical Society of America, 27, 132-137.

Traunmüller, H. (1981). Perceptual dimension of openness in vowels. Journal of the Acoustical Society of America, 69, 1465-1475.

Traunmüller, H. (1990). Analytical expressions for the tonotopic sensory scale. Journal of the Acoustical Society of America, 88, 97 100.

Vilkman, E., Aaltonen, O., Raimo, I., Arajävi, P., \& Oksanen, H. (1989). Articulatory hyoid-laryngeal changes versus cricothyroid activity in the control of intrinsic $F_{0}$ of vowels. Journal of Phonetics, 17, 193-203.

Whalen, D. H., Levitt, A. G., Hsiao, P.-L., \& Smorodensky, I. (1995). Intrinsic $F_{0}$ of vowels in the babbling of 6-, 9-, and 12 month-old French- and English-learning infants. Journal of the Acoustical Society of America, 97, 2533-2539.

Zwicker, E., \& FELDTKeller, R. (1967). Das Ohr als Nachrichtenempfänger [The ear as an information receiver] (2nd ed., rev.). Stuttgart: S. Hirzel.

\section{NOTES}

1. The phonetic "height" dimension was originally so called because it was thought that vowels of different height were made by raising or lowering the tongue body. In English, there are two series of monophthongal vowels varying in height, one made with the tongue body near the front of the mouth (front vowels) and one with the tongue body near the back of the mouth (back vowels). The front vowel series, in order of descending height, are those in beet, bit, bet, and back; the back vowels are those in boot, book, bought, and father.

2 . The term excitation pattern will be used to refer to the pattern of activity along the tonotopically organized basilar membrane created by a particular sound. Because basilar membrane activity is also reflected in the discharge rates of neurons in the auditory nerve, which is also tonotopically organized, the term could equally apply to the auditory nerve response. The term is intended here to indicate that representation of spectra originates in processes occurring in the basilar membrane, not to suggest that the brain directly inspects the basilar membrane in order to perceive sounds.

3. Since our performing the present study, Traunmüller and Syrdal have indicated to us (both by personal communication, February 20 , 1994) that they did not intend their studies to be interpreted in terms of simple place coding of frequency, with $F_{0}$ being represented by $\mathrm{H} 1$. However, as we have shown in the text, this was not made clear in the published papers. The interpretations suggested by Traunmüller and Syrdal in their personal communications are treated in the general discussion.

4. The equations used were those described by Traunmüller (1990):

$$
\begin{array}{ll}
z=[28.81 f /(1960+f)]-0.53 & (\text { for } f>200 \mathrm{~Hz}), \\
f=1960(z+0.53) /(26.28-z) & (\text { for } f>200 \mathrm{~Hz}),
\end{array}
$$

where $f=$ frequency in hertz and $z=$ frequency in Bark.

5. In the $100-\mathrm{Hz} F_{0}$ condition, two harmonics were filtered out, in the interests of using a common filter cutoff for all $F_{0}$ series. Thus the lowest frequency present in the stimuli in the filtered conditions was not always the second harmonic, nor was the lowest frequency perfectly correlated with $F_{0}$. However, for the purposes of the points being made, this can be overlooked (especially since there was very little difference between the 150 - and $125-\mathrm{Hz} F_{0}$ conditions)

(Manuscript received November 17, 1994, revision accepted for publication October 15, 1995.) 